\title{
An iterative method based on fractional derivatives for solving nonlinear equations
}

\author{
Béla J. Szekeres and Ferenc Izsák
}

\section{Introduction}

The theory of fractional order derivatives are almost as old as the integer-order [5]. There are many applications, for example in physics [1], [2], [6], finance [8], [9] or biology [3]. Our aim is to prove theoretical mathematical statements.

In this work our goal is to find a solution numerically for the equation $A(u)=f$. If we assume that $u$ is time-dependent, then one can do this by finding a stationary solution of the equation $\partial_{t} u(t)=-(A(u(t))-f)$. The numerical solution of this problem can be highly inaccurate. To avoid this we propose to replace the time derivative with a fractional one. Since the fractional order time derivative is a nonlocal operator, we expect that this stabilizes the time integration in the numerical solutions. Since the fractional order derivative here is defined as a limit of linear combination of past values, the time discretization will be simple. We also tested our method numerically in a fluid dynamical problem [10].

Béla J. Szekeres

Department of Numerical Analysis, Eötvös Loránd University, Faculty of Informatics, Pázmány P. stny. 1C, 1117 - Budapest, Hungary e-mail: szekeres@inf.elte.hu

Ferenc Izsák

Department of Applied Analysis and Computational Mathematics, \& MTA ELTE NumNet Research Group, Eötvös Loránd University, Pázmány P. stny. 1C, 1117 - Budapest, Hungary, e-mail: izsakf@cs.elte.hu 


\section{Mathematical preliminaries}

The following theorem is well known, see [11].

Theorem 1. Let $H$ real Hilbert-space, $A: H \rightarrow H$ nonlinear operator, which satisfies the conditions below with some positive constants $M \geq m$ :

1. $\langle A(u)-A(v), u-v\rangle \geq m\|u-v\|^{2}$,

2. $\|A(u)-A(v)\| \leq M\|u-v\|$.

Then for any $f, u_{0} \in H$ there exist a unique solution $u^{*}$ of the equation $A(u)=f$. If $t \in \mathbb{R}^{+}$is small enough the following iteration converges to $u^{*}$.

$$
u_{n+1}=u_{n}-t\left[A\left(u_{n}\right)-f\right] \text {. }
$$

There exist many different definitions of the fractional derivative [4], [7] we will use here the one below which is based on finite differences.

Definition 1. For the exponent $\beta \in(0,1)$ the fractional order derivative for a given function $f: \mathbb{R}^{+} \rightarrow \mathbb{R}$ is defined as

$$
\frac{\partial^{\beta} f(t)}{\partial t^{\beta}}:=\lim _{N \rightarrow \infty}\left\{\sum_{k=0}^{N}\left(\begin{array}{l}
\beta \\
k
\end{array}\right)(-1)^{k} \frac{f(t-k h)}{h^{\beta}}\right\},
$$

provided that the limit exists.

\section{Results}

Shortly, our objective is to find a solution for the equation $A(u)=f$ for a given nonlinear operator $A$, and for a given function $f$. The solution $u$ is also time-dependent, our goal is to find a stationary solution for

$$
-(A(u(t))-f)=\partial_{t} u(t)
$$

The method in Theorem 1 is one approach to this. Our idea was that to replace the time derivative in (2) with $\frac{\partial^{\beta}}{\partial t^{\beta}}$ for some $\beta \in(0,1)$, according to Definition 1 , and discretise the equation in time by a natural way.

We need an additional statement before we prove.

Lemma 1. (Pachpatte) Let $\left(\alpha_{n}\right)_{n \in \mathbb{N}},\left(f_{n}\right)_{n \in \mathbb{N}},\left(g_{n}\right)_{n \in \mathbb{N}},\left(h_{n}\right)_{n \in \mathbb{N}}$ nonnegative real sequences with the conditions below:

$$
\alpha_{n} \leq f_{n}+g_{n} \sum_{s=0}^{n-1} h_{s} \alpha_{s}
$$

Then the following inequality holds 


$$
\alpha_{n} \leq f_{n}+g_{n} \sum_{s=0}^{n-1} h_{s} f_{s} \prod_{\tau=s+1}^{n-1}\left(h_{\tau} g_{\tau}+1\right) .
$$

The main result is a generalisation of Theorem 1. For simplicity, we will not prove the existence of the solution.

Theorem 2. Let $H$ be real Hilbert-space, $A: H \rightarrow H$ a nonlinear operator, which satisfies the conditions below with some positive constants $M \geq m$ :

1. $\langle A(u)-A(v), u-v\rangle \geq m\|u-v\|^{2}$,

2. $\|A(u)-A(v)\| \leq M\|u-v\|$.

Let $u^{*}$ denote the solution of the equation $A(u)=f$. For any $f, u_{0} \in H \alpha \in(0,1)$, and $t \in \mathbb{R}^{+}$small enough the following iteration converges to $u^{*}$.

$$
u_{n+1}=\sum_{j=1}^{n+1}\left(\begin{array}{c}
\alpha \\
j
\end{array}\right)(-1)^{j+1} u_{n+1-j}-t\left[A\left(u_{n+1}\right)-f\right] .
$$

Proof. We first add $t\left[A\left(u_{n+1}\right)-f\right]-u^{*}$ both sides of the equation (5) and taking their norms, we have that

$$
\left\|u_{n+1}-u^{*}+t\left[A\left(u_{n+1}\right)-A\left(u^{*}\right)\right]\right\|=\left\|\sum_{j=1}^{n+1}\left(\begin{array}{c}
\alpha \\
j
\end{array}\right)(-1)^{j+1} u_{n+1-j}-u^{*}\right\| .
$$

Using the first assumption, we get the lower estimation

$$
\begin{array}{r}
\left\|u_{n+1}-u^{*}+t\left[A\left(u_{n+1}\right)-A\left(u^{*}\right)\right]\right\|^{2} \\
=\left\|u_{n+1}-u^{*}\right\|^{2}+t^{2}\left\|A\left(u_{n+1}\right)-A\left(u^{*}\right)\right\|^{2}+2 t\left\langle A\left(u_{n+1}\right)-A\left(u^{*}\right), u_{n+1}-u^{*}\right\rangle \\
\geq\left\|u_{n+1}-u^{*}\right\|^{2}+2 t m\left\|u_{n+1}-u^{*}\right\|^{2} \geq\left\|u_{n+1}-u^{*}\right\|^{2} .
\end{array}
$$

It is also known that $\sum_{j=1}^{\infty}\left(\begin{array}{c}\alpha \\ j\end{array}\right)(-1)^{j+1}=1$ and $\left(\begin{array}{c}\alpha \\ j\end{array}\right)(-1)^{j+1}>0$. Using this, the triangle inequality and (6) for the inequality in (7) we get

$$
\begin{array}{r}
\left\|u_{n+1}-u^{*}\right\| \leq\left\|\sum_{j=1}^{n+1}\left(\begin{array}{c}
\alpha \\
j
\end{array}\right)(-1)^{j+1} u_{n+1-j}-u^{*}\right\| \\
=\left\|\sum_{j=1}^{n+1}\left(\begin{array}{c}
\alpha \\
j
\end{array}\right)(-1)^{j+1} u_{n+1-j}-\sum_{j=1}^{\infty}\left(\begin{array}{c}
\alpha \\
j
\end{array}\right)(-1)^{j+1} u^{*}\right\| \\
\leq \sum_{j=1}^{n+1}\left(\begin{array}{c}
\alpha \\
j
\end{array}\right)(-1)^{j+1}\left\|u_{n+1-j}-u^{*}\right\|+\sum_{j=n+2}^{\infty}\left(\begin{array}{c}
\alpha \\
j
\end{array}\right)(-1)^{j+1}\left\|u^{*}\right\| .
\end{array}
$$

Let $\alpha_{n}:=\left\|u_{n}-u^{*}\right\|, f_{n}:=\sum_{j=n+1}^{\infty}\left(\begin{array}{c}\alpha \\ j\end{array}\right)(-1)^{j+1}\left\|u^{*}\right\|$ and $\beta_{n}=\left(\begin{array}{c}\alpha \\ n\end{array}\right)(-1)^{n+1}$. With these, we can rewrite (8) as 


$$
\alpha_{n+1} \leq f_{n+1}+\sum_{j=1}^{n+1} \beta_{j} \alpha_{n+1-j}
$$

Also using the notation $h_{j}$ instead of $\beta_{n+1-j}$, (9) can be recognised as

$$
\alpha_{n+1} \leq f_{n+1}+\sum_{j=0}^{n} h_{j} \alpha_{j}
$$

Therefore, with $g_{n}:=1$ we can apply Lemma 1 .

$$
\alpha_{n+1} \leq f_{n+1}+\sum_{s=0}^{n} h_{s} f_{s} \prod_{\tau=s+1}^{n}\left(h_{\tau}+1\right) .
$$

Estimate $\prod_{\tau=s+1}^{n}\left(h_{\tau}+1\right)$ as

$$
\begin{array}{r}
\prod_{\tau=s+1}^{n}\left(h_{\tau}+1\right)=\prod_{\tau=s+1}^{n}\left(\beta_{n+1-\tau}+1\right) \\
\leq \prod_{\tau=1}^{n}\left(\beta_{n+1-\tau}+1\right) \leq\left(\frac{n+\sum_{j=1}^{n} \beta_{j}}{n}\right)^{n} \leq\left(1+\frac{1}{n}\right)^{n} \leq e .
\end{array}
$$

Consequently, for (11) the following holds.

$$
\alpha_{n+1} \leq f_{n+1}+\sum_{s=0}^{n} h_{s} f_{s} \prod_{\tau=s+1}^{n}\left(h_{\tau}+1\right) \leq f_{n+1}+e \sum_{s=0}^{n} h_{s} f_{s} .
$$

It is clear that if $n \rightarrow \infty$ then $f_{n+1} \rightarrow 0$. We prove that $\sum_{s=0}^{n} h_{s} f_{s} \rightarrow 0$.

$$
\begin{array}{r}
\sum_{s=0}^{n} h_{s} f_{s}=\left\|u^{*}\right\| \beta_{n+1}+\left\|u^{*}\right\| \sum_{s=1}^{n} \beta_{n+1-s} \sum_{j=s+1}^{\infty} \beta_{j} \\
=\left\|u^{*}\right\| \beta_{n+1}+\left\|u^{*}\right\| \sum_{s=1}^{n} \beta_{n+1-s}\left(1-\sum_{j=1}^{s} \beta_{j}\right) \\
=\left\|u^{*}\right\| \beta_{n+1}+\left\|u^{*}\right\| \sum_{s=1}^{n} \beta_{n+1-s}-\left\|u^{*}\right\| \sum_{s=1}^{n} \sum_{j=1}^{n} \beta_{n+1-s} \beta_{j} .
\end{array}
$$

Observe first, that the last term in (12) is a Cauchy product.

$$
\lim _{n \rightarrow \infty}\left(\sum_{s=1}^{n} \sum_{j=1}^{n} \beta_{n+1-s} \beta_{j}\right)=\left(\sum_{j=1}^{\infty} \beta_{j}\right)^{2}=1 .
$$

Therefore, the first term in (12) tends to zero, the second and the third term to $\left\|u^{*}\right\|$, since $\sum_{j=1}^{\infty} \beta_{j}=1$. This means that $\alpha_{n+1} \rightarrow 0$ if $n \rightarrow \infty$, which has been stated. 


\section{Discussion}

In this work, we solved nonlinear time-independent equations of type $A(u)=f$, where the operator $A$ is on a Hilbert space. We assumed that it is monotone and Lipschitz-continuous and we proved that the algorithm is convergent.

Our numerical experiences show that if we replace the time-derivative operator in the equation $\partial_{t} u=-[A(u)-f]$ with a fractional derivative, then it stabilizes the time integration in the numerical solutions. We have tested our method numerically in a fluid dynamical problem previously [10].

Acknowledgements This work was completed in the ELTE Institutional Excellence Program (1783-3/2018/FEKUTSRAT) supported by the Hungarian Ministry of Human Capacities. The project has also been supperted by the European Union, co-financed by the Social Fund. EFOP3.6.1- 16-2016-0023.

\section{References}

1. Blumen, A., Zumofen, G., Klafter, J.: Transport aspects in anomalous diffusion: Lévy walks. Phys. Rev. A 40 (7), 3964-3973 (1989)

2. Bouchaud, J., Georges, A.: Anomalous diffusion in disordered media: Statistical mechanisms, models and physical applications. Physics Reports 195 (4), 127-293 (1990)

3. Edwards, A. M., Phillips, R. A., Watkins, N. W., Freeman, M. P., Murphy, E. J., Afanasyev, V., Buldyrev, S. V., da Luz, M. G. E., Raposo, E. P., Stanley, H. E., Viswanathan, G. M.: Revisiting Lévy flight search patterns of wandering albatrosses, bumblebees and deer. Nature 449, 1044-1048 (2007)

4. Kwaśnicki, M.: Ten equivalent definitions of the fractional laplace operator. Fractional Calculus and Applied Analysis 20 (1), 7-51 (2017)

5. Leibniz, G. W.: Mathematische Schriften. Georg Olms Verlagsbuchhandlung, Hildesheim (1962)

6. Metzler, R., Klafter, J.: The random walk's guide to anomalous diffusion: a fractional dynamics approach. Physics Reports 339 (1), 1-77 (2000)

7. Podlubny, I.: Fractional Differential Equations. Mathematics in Science and Engineering 198, Academic Press Inc., San Diego, CA (1999)

8. Sabatelli, L., Keating, S., Dudley, J., Richmond, P.: Waiting time distributions in financial markets. Physics of Condensed Matter 27, 273-275 (2002)

9. Scalas, E., Gorenflo, R., Mainardi, F.: Fractional calculus and continuous-time finance. Physica A: Statistical Mechanics and its Applications 284 (1), 376-384 (2000)

10. Szekeres, B. J., Izsák, F.: Fractional derivatives for vortex simulations. ALGORITMY 2016: 20th Conference on Scientific Computing Vysoké Tatry - Podbanské, Slovakia March 13 18, Slovak University of Technology in Bratislava, 175-182 (2016)

11. Zeidler, E.: Nonlinear Functional Analysis and its Applications: II/B: Nonlinear Monotone Operators. Springer-Verlag, New York (1990) 